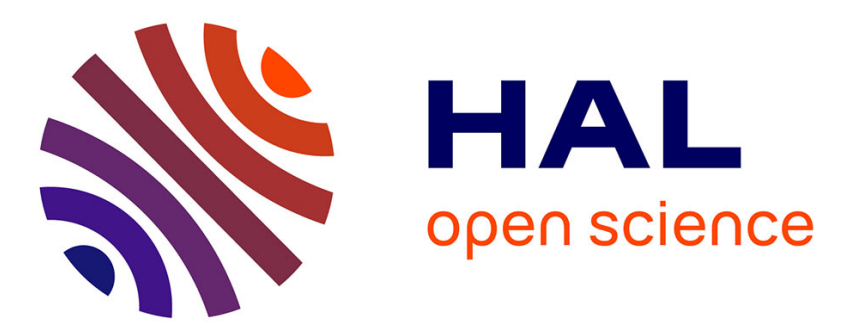

\title{
Optimisation et synthèse d'amplificateurs micro-ondes par la méthode des fréquences réelles
}

\author{
P. Jarry, A. Perennec, J. Le Bihan
}

\section{To cite this version:}

P. Jarry, A. Perennec, J. Le Bihan. Optimisation et synthèse d'amplificateurs micro-ondes par la méthode des fréquences réelles. Revue de Physique Appliquée, 1988, 23 (2), pp.137-142. 10.1051/rphysap:01988002302013700 . jpa-00245756

\section{HAL Id: jpa-00245756 https://hal.science/jpa-00245756}

Submitted on 1 Jan 1988

HAL is a multi-disciplinary open access archive for the deposit and dissemination of scientific research documents, whether they are published or not. The documents may come from teaching and research institutions in France or abroad, or from public or private research centers.
L'archive ouverte pluridisciplinaire HAL, est destinée au dépôt et à la diffusion de documents scientifiques de niveau recherche, publiés ou non, émanant des établissements d'enseignement et de recherche français ou étrangers, des laboratoires publics ou privés. 


\title{
Optimisation et synthèse d'amplificateurs micro-ondes par la méthode des fréquences réelles (*)
}

\author{
P. Jarry, A. Perennec et J. Le Bihan \\ Laboratoire d'Electronique et Systèmes de Télécommunications, UFR des Sciences et Techniques, 6 av. Le \\ Gorgeu, 29287 Brest Cedex, France
}

(Reçu le 16 janvier 1987, révisé le 13 novembre 1987, accepté le 19 novembre 1987)

\begin{abstract}
Résumé. - La méthode des «fréquences réelles » introduite par Carlin [1, 2] est basée uniquement sur la connaissance des paramètres de répartition $S_{i j}$ des transistors ; son utilisation ne nécessite aucun modèle de TEC. Le programme de CAO présenté utilise la procédure de Carlin. L'efficacité de la méthode, après modifications [3], permet aussi de concevoir des amplificateurs à plusieurs étages optimisés simultanément en gain et en TOS. Cette méthode tient compte de la non-unilatéralité des transistors.
\end{abstract}

\begin{abstract}
In many applications such as in communication or surveillance systems we require broadband microwave amplifiers. The purpose of this paper is to give simple and efficient broadband matching techniques for microwave transistors. The computer-aided design procedure utilizes only the measured scattering parameter of the FET devices (« real frequency » technique). The optimization process is carried simultaneously on the transducer power gain and the VSWR. Applications of the technique are given on a 8-12 GHz amplifier and a $19-21 \mathrm{GHz}$ amplifier.
\end{abstract}

\section{L'adaptation en fonction de la fréquence réelle.}

La méthode consiste à déterminer les admittances d'entrée des adaptateurs ou égaliseurs $E_{1}$ et $E_{2}$ (Fig. 1) :

$$
Y_{q_{1,2}}(j \omega)=G_{q_{1,2}}(\omega)+j B_{q_{1,2}}(\omega)
$$

à partir des admittances connues du transistor :

$$
Y_{L_{1,2}}(j \omega)=G_{L_{1,2}}(\omega)+j B_{L_{1,2}}(\omega) .
$$

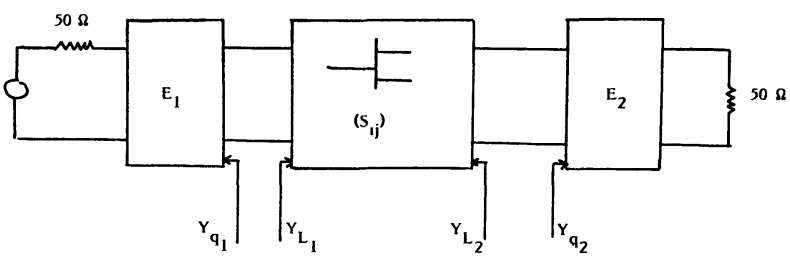

Fig. 1. - L'amplificateur et les deux adaptateurs d'entrée et de sortie.

[Amplifier with input and output equalizers.]

(*) Cet article avait été proposé pour le $\mathrm{N}^{\circ}$ spécial «Semiconducteurs III-V». Sa parution a été retardée pour des raisons matérielles indépendantes de l'éditeur.
Le gain transducique qui est le rapport de la puissance délivrée à la charge sur la puissance disponible au générateur peut se mettre sous la forme d'un produit de deux termes :

$$
T(\omega)=K_{1} \cdot K_{2}
$$

avec :

$$
K_{1}=\frac{\left|S_{21}(\omega)\right|}{1-\left|S_{11}(\omega)\right|^{2}} \cdot \frac{4 G_{q_{1}}(\omega) G_{L_{1}}(\omega)}{\left(G_{q_{1}}+G_{L_{1}}\right)^{2}+\left(B_{q_{1}}+B_{L_{1}}\right)^{2}} .
$$

Le terme de droite rend compte de l'adaptation égaliseur-transistor. L'optimisation consistera ici à rendre $K_{1}$ aussi voisin que possible d'une constante $K_{10}$ dans la bande passante.

L'expression de $K_{2}$ est du même type après avoir fait le changement $S_{11}$ en $S_{2}=S_{22}+S_{12} S_{21} S_{q_{1}}$ $\left(1-S_{11} S_{q_{1}}\right)^{-1}$ où $S_{q_{1}}$ est le coefficient de réflexion associé à $Y_{q_{1}}$.

L'originalité de la méthode consiste à représenter $G_{q_{1}}(\omega)$ par une suite de segments de droite décrits 
par des fonctions $a_{k}\left(\omega_{j}\right)$ et un vecteur $\mathbf{r}$

$$
\begin{aligned}
G_{q_{1}}\left(\omega_{j}\right) & =\sum_{k=0}^{N} a_{k}\left(\omega_{j}\right) r_{k}=\mathbf{a}^{T} \mathbf{r} \\
\text { avec } a_{k}\left(\omega_{j}\right) & =\left[\begin{array}{lll}
1 & \text { si } \omega_{j}>\omega_{k} \\
\frac{\omega_{j}-\omega_{k-1}}{\omega_{k}-\omega_{k-1}} & \text { si } \omega_{k-1}<\omega_{j}<\omega_{k} \\
0 & \text { si } \omega_{j}<\omega_{k-1}
\end{array}\right.
\end{aligned}
$$

$\omega_{k-1}$ et $\omega_{k}$ définissent les bornes du segment.

Les $\omega_{j}$ sont les fréquences réelles de mesure des paramètres $S_{i j}$ du transistor. L'égaliseur $\mathrm{E}_{1}$ étant à phase minimum, $B_{q_{1}}$ est la transformée de Hilbert de $G_{q_{1}}$ et s'écrit alors $B_{q_{1}}=\mathbf{b}^{T} \mathbf{r}$. Les $r_{k}$ deviennent donc les inconnues du problème d'adaptation, en effet :

$$
\begin{aligned}
& \qquad \begin{aligned}
K_{1} & =W_{1}\left(\omega_{j}\right) \cdot \frac{4 G_{L_{1}} \mathbf{a}^{T} \mathbf{r}}{\left(\mathbf{a}^{T} \mathbf{r}+G_{L_{1}}\right)^{2}+\left(\mathbf{b}^{T} \mathbf{r}+B_{L_{1}}\right)^{2}} \\
\text { soit } K_{1} & =W_{1}\left(\omega_{j}\right) \cdot T_{1}\left(\mathbf{r}, \omega_{j}\right)
\end{aligned}
\end{aligned}
$$

avec

$$
W_{1}\left(\omega_{j}\right)=\frac{\left|S_{21}\left(\omega_{j}\right)\right|}{1-\left|S_{11}\left(\omega_{j}\right)\right|^{2}}
$$

Dans le processus d'optimisation, cette quantité $W_{1}\left(\omega_{j}\right)$ sera considérée comme une fonction poids.

Bien entendu $K_{2}$ a une forme identique avec une fonction de poids $W_{2}\left(\omega_{j}\right)$.

OPTIMISATION DU GAIN. - Le vecteur r sera calculé de manière à obtenir un gain plat sur une bande de fréquence $\left[\omega_{0}, \omega_{N_{1}}\right]$. La fonction objectif utilisée est du type moindres carrés. Soit pour l'égaliseur d'entrée

$$
E(\mathbf{r})=\sum_{j=0}^{N_{1}}\left[W_{1} \cdot T_{1}\left(\omega_{j}\right) / K_{1}-1\right]^{2}=\mathbf{e}^{T}(r) \mathbf{e}(r) .
$$

La minimisation de cette fonction d'erreur se fait par la méthode de Marquardt en utilisant l'algorithme décrit par More [4] qui assure une convergence efficace. La base de la méthode de Marquardt consiste à rajouter une constante $\alpha$ dans l'expression donnant la variation $\mathbf{p}$ de $\mathbf{r}\left(\mathbf{r}=\mathbf{r}_{0}+\mathbf{p}\right)$ qui minimise l'erreur quadratique. On a alors :

$$
\mathbf{p}=\left(\mathbf{J}^{T} \mathbf{J}+\alpha \mathbf{D}^{T} \mathbf{D}\right)^{-1} \mathbf{J}^{T} \mathbf{e}_{0}
$$

avec :

$\mathbf{J}$ : jacobien de et $\mathbf{e}_{0}$ : vecteur erreur initial

D : matrice diagonale servant de contrainte et fixant l'ordre de grandeur des composantes de p

$\alpha$ : paramètre de Levenberg-Marquardt.

L'algorithme de Moré permet l'ajustement de $\alpha$ et de $\mathbf{D}$ de manière adaptative. Il est basé sur l'analyse de la linéarité de la fonction $E(\mathbf{r})$ au voisinage du point courant à l'aide de la fonctionn :

$$
\rho(\mathbf{p})=\frac{\|E(\mathbf{r})\|^{2}-\|E(\mathbf{r}+\mathbf{p})\|^{2}}{\|E(\mathbf{r})\|^{2}-\|E(\mathbf{r})+\mathbf{J p}\|^{2}}
$$

où $\|E\|$ désigne la norme du vecteur.

La contrainte sur $\mathbf{p}$ consiste à satisfaire l'inégalité suivante :

$$
\|\mathbf{D p}\|<\Delta
$$

où $\Delta$ est une constante strictement positive ajustée à chaque itération suivant la valeur de $\rho(\mathbf{p})$.

SYNTHÈSE DES ÉGALISEURS [5]. - L'étape de synthèse consiste à remplacer les $G_{q_{1,2}}(\omega)$ par des fractions rationnelles réalisables sous forme de réseaux en échelles (par exemple des filtres passebas LC) :

$$
\hat{G}_{q_{1,2}}(\omega)=\frac{A_{0}}{B_{0}+B_{1} \omega^{2}+\cdots+B_{n} \omega^{2 n}} .
$$

L'approximation (c'est-à-dire la détermination de $A_{0}$ et des $B_{i}$ ) utilise le même sous-programme d'optimisation (Marquardt-More). Les égaliseurs $E_{1}$ et $E_{2}$ sont alors synthétisés par la méthode de Darlington [6] à partir des admittances $Y_{q_{1,2}}(p)$ rendues positives réelles (c'est-à-dire réalisables). La figure 2 montre l'organigramme de conception

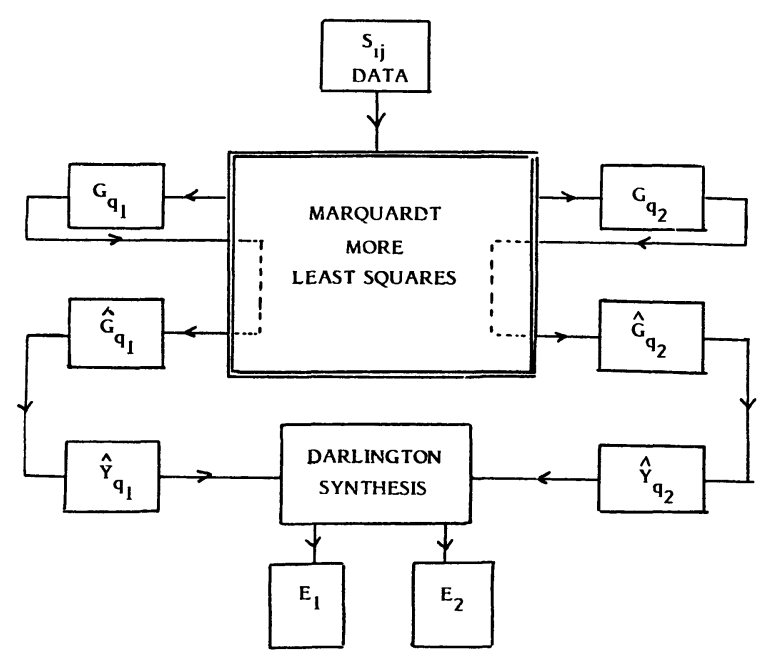

Fig. 2. - Organigramme de conception des égaliseurs d'entrée et de sortie.

[Flowchart of the routine conception of the input and output equalizers.]

des égaliseurs à partir des données d'entrée $\left(S_{i j}\right.$ du transistor). On peut voir sur la figure 3 les résultats obtenus dans le cas d'un transistor à effet de champ (les éléments série des égaliseurs sont des selfs données en $n H$ et les éléments parallèles des capacités données en $p F$ ) :
Générateur
$Z_{\mathrm{G}}=50 \Omega$
Charge
$Z_{\mathrm{L}}=50 \Omega$

Bande passante $8 \mathrm{GHz}<f<12 \mathrm{GHz}$

Elément actif FET (source Thomson). 


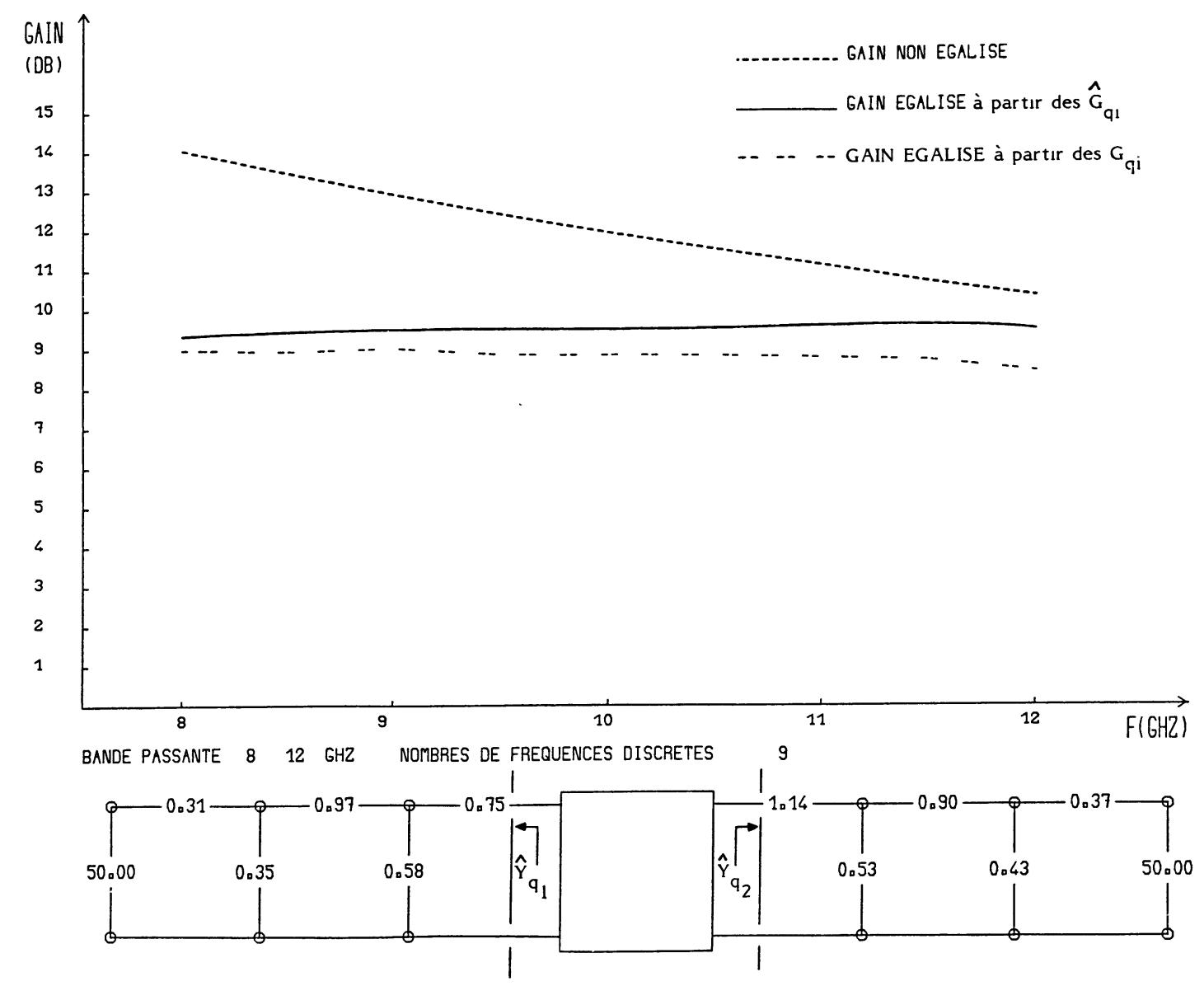

Fig. 3. - Gain optimisé d'un amplificateur ne comportant qu'un seul étage (8-12 GHz).

[Gain response of a $8-12 \mathrm{GHz}$ equalized FET amplifier.]

Ses paramètres de répartition $S_{i j}$ sont donnés dans le tableau I.

Tableau 1. - Paramètres de répartition mesurés (FET, source Thomson).

\begin{tabular}{ccccccccc}
\hline \multirow{2}{*}{$(\mathrm{GHz})$} & \multicolumn{2}{c}{$S_{11}$} & \multicolumn{2}{c}{$S_{12}$} & \multicolumn{2}{c}{$S_{21}$} & \multicolumn{2}{c}{$S_{22}$} \\
& module & phase & module & phase & module & phase & module & phase \\
\hline 8 & 0,822 & $-103,0$ & 0,115 & 24,0 & 2,45 & 102,0 & 0,517 & $-56,2$ \\
8,5 & 0,814 & $-107,0$ & 0,118 & 21,6 & 2,35 & 98,4 & 0,510 & $-58,5$ \\
9,0 & 0,806 & $-110,0$ & 0,120 & 19,4 & 2,26 & 95,4 & 0,504 & $-60,8$ \\
9,5 & 0,800 & $-114,0$ & 0,121 & 17,2 & 2,18 & 92,5 & 0,498 & $-63,0$ \\
10,0 & 0,794 & $-117,0$ & 0,123 & 15,2 & 2,10 & 89,7 & 0,494 & $-65,1$ \\
10,5 & 0,788 & $-120,0$ & 0,124 & 13,2 & 2,02 & 87,0 & 0,490 & $-67,3$ \\
11,0 & 0,783 & $-122,0$ & 0,125 & 11,3 & 1,95 & 84,4 & 0,486 & $-69,4$ \\
11,5 & 0,778 & $-125,0$ & 0,126 & 9,56 & 1,88 & 81,8 & 0,483 & $-71,4$ \\
12,0 & 0,774 & $-127,0$ & 0,127 & 7,84 & 1,82 & 79,3 & 0,481 & $-73,5$
\end{tabular}

En suivant les différentes étapes indiquées sur ia figure 2 , on obtient $\hat{G}_{q_{1}}$ et $\hat{G}_{q_{2}}$ sous la forme suivante :

$$
\begin{aligned}
& \hat{G}_{q_{1}}=\frac{1}{1-1,08 \omega^{2}+1,05 \omega^{4}-0,15 \omega^{6}-0,24 \omega^{8}+0,09 \omega^{10}} \\
& \hat{G}_{q_{2}}=\frac{1}{1-1,62 \omega^{2}+3,59 \omega^{4}-0,77 \omega^{6}-0,87 \omega^{8}+0,31 \omega^{10}}
\end{aligned}
$$


puis les admittances correspondantes $Y_{q_{1}}$ et $Y_{q_{2}}$

$$
\begin{aligned}
& \hat{Y}_{q_{1}}=\frac{1+2,336 p+2,144 p^{2}+1,250 p^{3}+0,396 p^{4}+0 p^{5}}{1+2,038 p+2,616 p^{2}+1,882 p^{3}+0,937 p^{4}+0,297 p^{5}} \\
& \hat{Y}_{q_{2}}=\frac{1+2,417 p+2,113 p^{2}+1,305 p^{3}+0,489 p^{4}+0 p^{5}}{1+2,427 p+3,754 p^{2}+2,791 p^{3}+1,502 p^{4}+0,561 p^{5}} .
\end{aligned}
$$

Le gain obtenu pour l'ensemble FET-égaliseurs est compris entre 9 et $10 \mathrm{~dB}$.

Cette méthode simple qui donne entière satisfaction est néanmoins limitée au cas d'un seul transistor. La mise en cascade de plusieurs étapes se fera en utilisant la méthode des «fréquences réelles simplifiées » [3].

\section{Amplificateurs comportant plusieurs étages.}

Le gain en puissance des $k$ premiers étages de l'amplificateur de la figure 4 est donné par [3]:

$$
T_{k}(\omega)=T_{(k-1)} \frac{\left|e_{21 k}\right|^{2}\left|S_{21 k}\right|^{2}}{\left|1-e_{11 k} S_{G k}\right|^{2}\left|1-\hat{e}_{22 k} S_{11 k}\right|^{2}}
$$
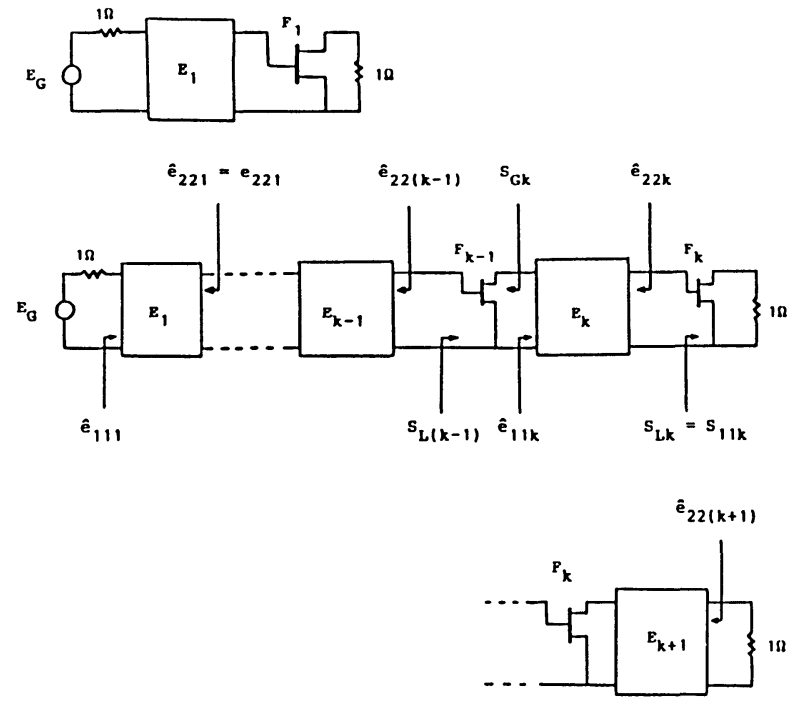

Fig. 4. - Les différentes étapes pour la conception des amplificateurs à plusieurs étages.

[Computation steps for designing broad-band multistage microwave FET amplifiers.]

soit

$$
T_{k}(\omega)=T_{(k-1)} \cdot E_{k}(\omega)
$$

où

$T_{k-1}$ : gain en puissance des $(k-1)$ premiers étages

$\left(e_{i j}\right)_{k}$ : paramètres de répartition du $k$-ième égaliseur $\mathrm{E}_{k}$

$\left(S_{i j}\right)_{k}$ : paramètres de répartition du $k$-ième FET $\left(\mathrm{F}_{k}\right)$ $\hat{e}_{22 k}$ : coefficient de réflexion à la sortie de $\mathrm{E}_{k}$ vers le générateur

$S_{\mathrm{G} k}$ : coefficient de réflexion à la sortie de $\mathrm{F}_{k-1}$ vers le générateur.

Alors :

$$
\begin{aligned}
\hat{e}_{22 k} & =e_{22 k}+\frac{e_{21 k}^{2} S_{\mathrm{G} k}}{1-e_{11 k} S_{\mathrm{G} k}} \\
S_{\mathrm{G} k} & =S_{22(k-1)}+\frac{\left.S_{12(k-1)} S_{21(k-1)} \hat{e}_{22(k-1)}\right)}{1-S_{11(k-1)} e_{22(k-1)}} .
\end{aligned}
$$

Le gain en puissance total est défini après avoir placé le dernier égaliseur $E_{k+1}$

$$
T(\omega)=\left(T_{1}, T_{2} \ldots T_{k}\right) E_{k+1}(\omega) .
$$

De même nous pouvons donner l'expression du taux d'ondes stationnaires (TOS) de l'amplificateur multi-étages.

Conformément à la figure 4 le TOS d'entrée correspondant aux $k$ premiers étages est :

$$
R_{\mathrm{in} k}=\left.\frac{1+\left|\hat{e}_{111}\right|}{1-\left|\hat{e}_{111}\right|}\right|_{k}
$$

$R_{\text {ink }}$ est alors calculé de manière récursive à partir de la charge par:

$$
\begin{aligned}
S_{\mathrm{L} k} & =S_{11 k} \\
\hat{e}_{11 k} & =e_{11 k}+\frac{e_{21 k}^{2} S_{\mathrm{L} k}}{1-e_{22 k} S_{\mathrm{L} k}} \\
S_{\mathrm{L}(k-1)} & =S_{11(k-1)}+\frac{S_{12(k-1)} S_{21(k-1)} \hat{e}_{11 k}}{1-S_{22(k-1)} \hat{e}_{11 k}}
\end{aligned}
$$

où

$\hat{e}_{11 k}$ : est le coefficient de réflexion mesuré à l'entrée de $\mathrm{E}_{k}$ vers la charge

$S_{\mathrm{L} k}$ : est le coefficient de réflexion mesuré à l'entrée de $F_{k}$ vers la charge.

Après avoir placé le dernier égaliseur $\mathrm{E}_{k+1}$ nous obtenons le TOS d'entrée de la chaîne complète $R_{\text {in }}$ ainsi que celui de la sortie $R_{\text {out }}$.

$$
\begin{aligned}
R_{\mathrm{in}} & =R_{\mathrm{in}(k+1)}=\left.\frac{1+\left|\hat{e}_{111}\right|}{1-\left|\hat{e}_{111}\right|}\right|_{k+1} \\
R_{\mathrm{out}} & =\frac{1+\left|\hat{e}_{22(k+1)}\right|}{1-\left|\hat{e}_{22(k+1)}\right|} .
\end{aligned}
$$


OPTIMISATION DU GAIN ET DU TAUX D'ONDES STATIONNAIRES. - L'égaliseur $\mathrm{E}_{k}$ est un quadripôle réciproque et non dissipatif. Conformément à la représentation de Belevitch [7], les éléments de sa matrice de répartition peuvent se mettre sous la forme :

$$
\begin{aligned}
& e_{11 k}=h(p) / g(p) \\
& e_{12 k}=e_{21 k}(p)= \pm p^{\ell} / g(p) \\
& e_{22 k}=-(-1)^{\ell} h(-p) / g(p)
\end{aligned}
$$

$h(p)$ et $g(p)$ étant des polynômes de degré $n$ et $\ell \geqslant 0$ un entier spécifiant l'ordre du zéro de transmission (c'est-à-dire de $e_{12}(p)$ ) pour $p$ infini. Par la suite les coefficients $h_{i}$ de $h(p)=h_{0}+h_{1} p+\cdots+h_{n} p^{n}$, seront pris comme inconnues ; $g(p)$ sera alors déterminée dans les étapes d'optimisation et de synthèse après factorisation de la relation de non-dissipativité de $\mathrm{E}_{k}$ :

$$
g(p) g(-p)=h(p) h(-p)+(-1)^{\ell} p^{2 \ell} .
$$

Le gain en puissance et le TOS sont optimisés simultanément et étage par étage de la manière suivante :

1) Le premier égaliseur $E_{1}$ permet d'adapter le gain $T_{1}(\omega)=\mathrm{E}_{1}(\omega)$ et le TOS d'entrée $R_{\text {in } 1}$ du premier transistor $F_{1}$ fermé sur $1 \Omega$. La fonction objectif est alors de la forme :

$$
\begin{aligned}
u=\sum_{i=1}^{m}\left\{W_{1}\left(\frac{T_{1}\left(\omega_{i}\right)}{T_{10}}-1\right)^{2}\right. & + \\
& \left.+W_{2}\left(\frac{R_{\text {in } 1}\left(\omega_{i}\right)}{R_{\text {in } 0}}-1\right)^{2}\right\} .
\end{aligned}
$$

La minimisation de la fonction objectif du type moindres carrés se fait en utilisant l'algorithme de Marquardt-More déjà décrit ; les coefficients $h_{i}+\Delta h_{i}$ étant corrigés à chaque itération.

2) Le second étage (égaliseur $\mathrm{E}_{2}$ plus transistor $F_{2}$ fermé sur $1 \Omega$ ) est placé en cascade. Ce second égaliseur permet d'optimiser simultanément le gain $T_{2}(\omega)=T_{1} \cdot \mathrm{E}_{2}(\omega)$ et le TOS d'entrée $R_{\text {in } 2}$ de l'ensemble. La quantité $T_{1}$ est considérée comme une fonction de poids.

3) Tous les étages sont mis en cascade suivant la procédure $n^{\circ} 2$. Seul le dernier égaliseur $\mathrm{E}_{k+1}$ est utilisé pour adapter le TOS de sortie $R_{\text {out }}$.

La figure 5 montre l'organigramme de conception des égaliseurs à partir des paramètres $S_{i j}$ du transistor.

Chaque égaliseur étant réalisé sous la forme d'un réseau passe-bas en échelle, l'exposant $\ell$ et le coefficient $h(0)$ sont nuls pour chacune des fonctions $e_{11}(p)$.

On peut voir sur la figure 6 les résultats obtenus

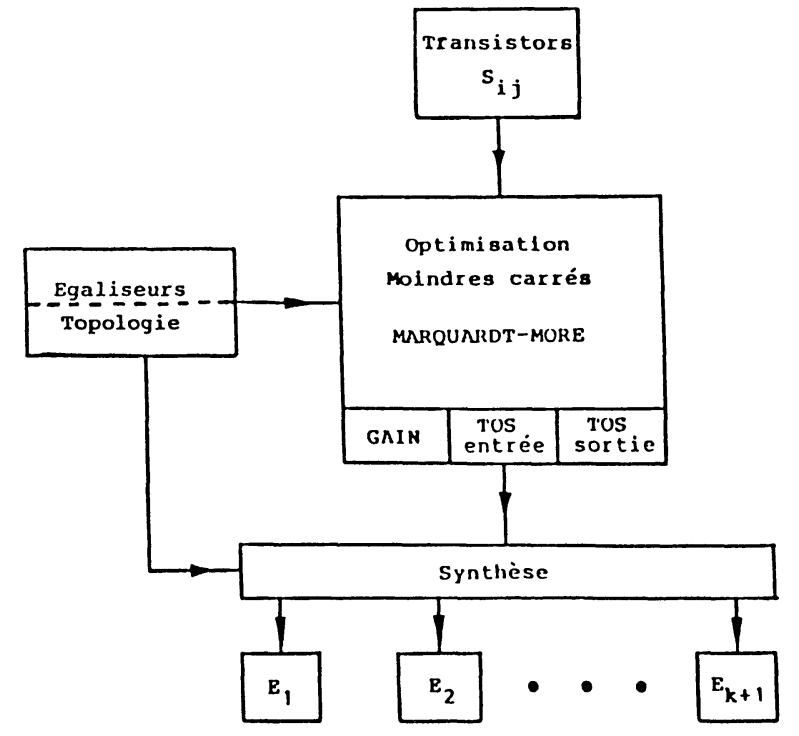

Fig. 5. - Organigramme de conception des amplificateurs microondes à plusieurs étages.

[Flowchart of the routine conception for multistage microwave amplifiers.]

dans le cas d'un amplificateur micro-ondes à quatre transistors :

\section{Générateur $\quad S_{\mathrm{G} 1}=0\left(Z_{\mathrm{G}}=50 \Omega\right)$ \\ Charge $\quad S_{\mathrm{L}}=0\left(Z_{\mathrm{L}}=50 \Omega\right)$ \\ Bande passante $18 \mathrm{GHz}-21 \mathrm{GHz}$ \\ Eléments actifs 4 FET identiques (source CNET Lannion B).}

Leurs paramètres de répartition $S_{i j}$ sont donnés dans le tableau II.

Les égaliseurs d'entrée, inter-étages et de sortie sont synthétisés, étape par étape comme indiqué précédemment, à partir de leurs coefficients de réflexion $e_{11 i}(p)$ suivants obtenus par optimisation :

$$
\begin{aligned}
& e_{111}(p)=\frac{0-0,445 p-0,668 p^{2}}{1+1,29 p+0,668 p^{2}} \\
& e_{1{ }_{2}}(p)=\frac{0-0,287 p-0,484 p^{2}}{1+1,025 p+0,484 p^{2}} \\
& e_{113}(p)=\frac{0+0,903 p-0,251 p^{2}}{1+0,715 p+0,251 p^{2}} \\
& e_{114}(p)=\frac{0+0,136 p-0,248 p^{2}}{1+0,717 p+0,248 p^{2}} \\
& e_{115}(p)=\frac{0+0,271 p+0,480 p^{2}}{1+1,016 p+0,480 p^{2}} .
\end{aligned}
$$

Le gain de l'ensemble est de l'ordre de $20 \mathrm{db}$, le TOS d'entrée est inférieur à 2,3 et le TOS de sortie inférieur à 2,10 sur toute la bande. 


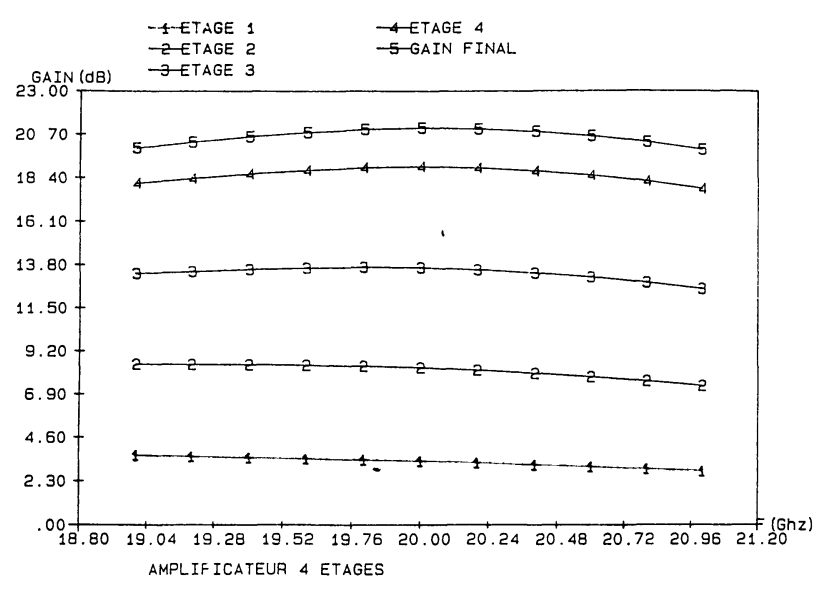

Fig. 6(a). - Gain optimisé d'un amplificateur à quatre étages $(19-21 \mathrm{GHz})$ : réponses des différents étages.

[Performance of the design steps of a four-stage amplifier (19-21 GHz).]

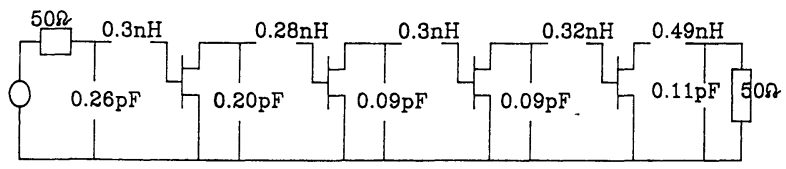

Fig. 6(c). - Topologie et conception de l'amplificateur à TEC (19-21 GHz).

[Design of the four-stage FET amplifier (19-21 GHz).]

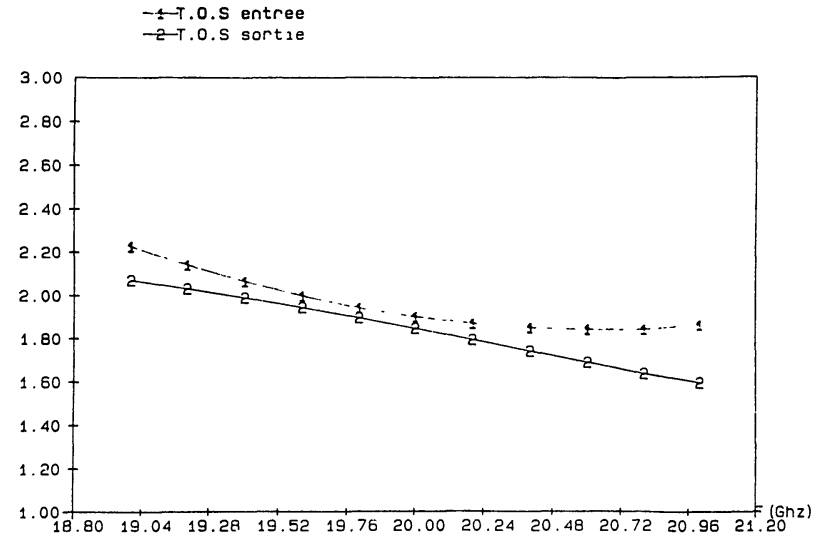

Fig. 6(b). - TOS d'entrée et de sortie de l'amplificateur microonde à quatre étages $(19-21 \mathrm{GHz})$.

[Input and output VSWR of the four-stage amplifier (19$21 \mathrm{GHz})$.]

\section{Conclusion.}

La méthode dite des «fréquences réelles » permet de concevoir et d'optimiser des amplificateurs micro-ondes à un ou plusieurs étages. Cette procédure est basée uniquement sur la connaissance des paramètres de répartition des transistors utilisés; elle ne nécessite donc pas d'utilisation d'un modèle ou d'un schéma équivalent des transistors. Elle permet aussi de tenir compte de la non-unilatéralité des transistors.

Tableau II. - Paramètres de répartition mesurés (FET, source CNET).

\begin{tabular}{ccccccccc}
\hline \multirow{2}{*}{ (GHz) } & \multicolumn{2}{c}{$S_{11}$} & \multicolumn{2}{c}{$S_{12}$} & \multicolumn{2}{c}{$S_{21}$} & \multicolumn{2}{c}{$S_{22}$} \\
& module & phase & module & phase & module & phase & module & phase \\
\hline 18 & $7,21 \mathrm{E}-01$ & $-143,0$ & $7,52 \mathrm{E}-02$ & 7,9 & $1,16 \mathrm{E}+00$ & 57,6 & $5,59 \mathrm{E}-01$ & $-73,9$ \\
19 & $7,19 \mathrm{E}-01$ & $-144,7$ & $7,52 \mathrm{E}-02$ & 6,8 & $1,10 \mathrm{E}+00$ & 54,4 & $5,68 \mathrm{E}-01$ & $-76,2$ \\
20 & $7,17 \mathrm{E}-01$ & $-146,2$ & $7,52 \mathrm{E}-02$ & 5,8 & $1,05 \mathrm{E}+00$ & 51,4 & $5,78 \mathrm{E}-01$ & $-78,4$ \\
21 & $7,16 \mathrm{E}-01$ & $-147,6$ & $7,52 \mathrm{E}-02$ & 4,9 & $9,96 \mathrm{E}-01$ & 48,4 & $5,88 \mathrm{E}-01$ & $-80,6$
\end{tabular}

\section{Bibliographie}

[1] CARlin, H. J., "A New Approach to gain-bandwith problems »IEEE Trans. CAS-23 (1977) 170.

[2] Carlin, H. J. and Amstutz, P., "On optimum broad-band matching 》 IEEE Trans. CAS-28 (1981) 401-405.

[3] Yarman, B. S. and Carlin, H. J., "A simplified «Real Frequency» Technique Applied to broad-band multi-stage microwave amplifiers » IEEE Trans. MTT-30 (1982) 2216-2222.

[4] MoRE, J. J., in The Levenberg-Marquardt algorithm : implementation and theory, Lecture notes in Mathematics 630 (Springer Verlag) 1978.
[5] Perennec, A. and JARry, P., «Real Frequency broadband microwave matching using a modified Marquardt optimization » IEEE Melecon'87, 34th Congress on Electronics, March 1987, Rome.

[6] Scanlan, S. O., Levy, R., Circuit Theory (Oliver and Boyd) 1970.

[7] BELEVITCH, V., « Elementary application of the scattering formalism to network design "IRE Trans. Circuit Theory C'r-3 (1956). 